Bull. Austral. Math. Soc.

$22 \mathrm{A05}, 20 \mathrm{E} 10$

VOL. 57 (1998) [147-151]

\title{
HAUSDORFFNESS IN VARIETIES OF TOPOLOGICAL GROUPS
}

\author{
Carolyn E. McPhail
}

\begin{abstract}
A variety of topological groups $\mathfrak{V}$ is a class of (not necessarily Hausdorff) topological groups closed under the operations of forming subgroups, quotient groups and arbitrary products. It is well known that the class of groups underlying the topological groups contained in any variety of topological groups is a variety of groups. Much work on topological groups is restricted to Hausdorff topological groups and so it is relevant to know if the class of groups underlying Hausdorff topological groups in $\mathfrak{V}$ is a variety of groups. It is shown that this is not always the case. Indeed it is proved that this is not the case for an important proper class of varieties of topological groups.
\end{abstract}

\section{Preliminaries}

Recall that a class $\mathcal{V}$ of groups is said to be a variety of groups [9] if it is closed under the operations of forming subgroups, quotient groups and arbitrary products. Similarly, Morris [5] defined a class $\mathfrak{V}$ of topological groups to be a variety of topological groups if it is closed under the operations of forming subgroups (with the subspace topology), quotient topological groups and arbitrary products (with the Tychnoff product topology). (For a survey of varieties of topological groups see [8].) Much work on topological groups deals only with Hausdorff topological groups so it is important to point out that our attention is not restricted to Hausdorff topological groups.

For a topological group $G$, let $|G|$ denote the group obtained by $G$ by "forgetting" the topology. We call $|G|$ the group underlying $G$. Further, let $|\mathfrak{V}|$ denote the class of all groups underlying the topological groups contained in the variety of topological groups $\mathfrak{V}$. Note that $|\mathfrak{V}|$ is obviously a variety of groups. Finally, let $|\mathfrak{V}|_{2}$ denote the class of all groups that, with a Hausdorff topology, appear in $\mathfrak{V}$. It is of interest to know if the class of groups $|\mathfrak{V}|_{2}$ is always a variety of groups.

Received 13th May, 1997

I would like to thank Professor Sidney A. Morris for his support and guidance in the writing of this paper. I greatly appreciate his confidence in me but most of all his patience with me.

Copyright Clearance Centre, Inc. Serial-fee code: 0004-9729/98 \$A2.00+0.00. 


\section{An Important Example}

Central to the study of varieties of topological groups are the $T(m)$-groups, where $m$ is some cardinal number. The $T(m)$-groups were introduced in Morris [6].

Definition 2.1: (See [6, Section 4].) Let $m$ be some cardinal number. A topological group is said to be a $T(m)$-group if each neighbourhood of the identity contains a normal subgroup of index strictly less than $m$.

The inclusion of "normal" in Definition 2.1 did not appear in [6]. However, our definition of $T(m)$-group has in recent years been considered to be a more useful concept $[3]$.

REMARK. Let $G$ be a topological group. Then $G$ is a $T(m)$-group for every $m$ strictly greater than the cardinality of $G$ (denoted card $(G)$ ). In particular, let $G$ be a discrete group (or any NSS-group) [7] such as any Lie group, or any Banach space considered as a topological group); then $G$ is a $T(m)$-group if and only if card $(G)$ is strictly less than $m$. As subgroups, quotient groups and arbitrary products of $T(m)$-groups are $T(m)$-groups, we obtain the following proposition.

Proposit ION 2.2. (See [6, Theorem 4.2].) Let $\mathcal{V}$ be a variety of groups and for any infinite cardinal number $m$, let $\mathfrak{V}_{m}$ be the class of all $T(m)$-groups $G$ such that $|G| \in \mathcal{V}$. Then $\mathfrak{V}_{m}$ is a variety of topological groups. Further, for distinct infinite cardinal numbers $m$ and $n, \mathfrak{V}_{m} \neq \mathfrak{V}_{n}$.

We shall use $\mathfrak{T}_{m}$ to denote the variety of all $T(m)$-groups and $\mathfrak{A}_{m}$ to denote the variety of all Abelian $T(m)$-groups.

\section{3. $T\left(\aleph_{0}\right)$-GROUPS}

We shall see below that the variety of all $T\left(\aleph_{0}\right)$-groups, $\mathfrak{T}_{\aleph_{0}}$, has the property that $\left|\mathfrak{T}_{\aleph_{0}}\right|_{2}$ is not a variety of groups. This is the first such example.

Recall that a group is said to be residually finite [4] if it is isomorphic to a subgroup of a product of finite groups. This algebraic property characterises those groups that underlie Hausdorff topological groups contained in $\mathfrak{T}_{\aleph_{0}}$.

Lemma 3.1. Let $G$ be a group. Then $G$ admits a Hausdorff group topology which makes it a $T\left(\aleph_{0}\right)$-group if and only if $G$ is residually finite.

Proof: Let $G$ be a group that admits a Hausdorff group topology which makes it a $T\left(\aleph_{0}\right)$-group. Let $\left\{U_{i}: i \in I\right\}$ be the family of all neighbourhoods of the identity 1 in $G$. Then for each $U_{i}$, there exists a normal subgroup $N_{i}$ of $G$ such that $1 \in N_{i} \subseteq U_{i}$ and $\operatorname{card}\left(G / N_{i}\right)$ is finite. Further, let $\phi_{i}: G \rightarrow G / N_{i}$ be the canonical homomorphism from $G$ onto $G / N_{i}$ and let $\Phi: G \rightarrow \prod_{i \in I} G / N_{i}$ be given by $\Phi(g)=\prod_{i \in I} \phi_{i}(g)$. Since 
the topology on $G$ is Hausdorff, $\bigcap_{i \in I} U_{i}=\{1\}$ and so $\Phi$ is a one-to-one homomorphism from $G$ into a product of finite groups. Therefore, $G$ is residually finite.

Conversely, let $G$ be a residually finite group. Then there exists an index set $I$ and finite groups $F_{i}$ for each $i \in I$ such that $G$ is isomorphic to a subgroup of $\prod_{i \in I} F_{i}$. Now, for each $i \in I, F_{i}$ with the discrete topology is a Hausdorff $T\left(\aleph_{0}\right)$-group. Since products of Hausdorff $T\left(\aleph_{0}\right)$-groups and subgroups of Hausdorff $T\left(\aleph_{0}\right)$-groups are Hausdorff $T\left(\aleph_{0}\right)$-groups, then $G$ with the induced topology from $\prod_{i \in I} F_{i}$ is a Hausdorff $T\left(\aleph_{0}\right)$-group.

The main result for this section will also use the following lemma.

Lemma 3.2. Let $G$ be a non-trivial divisible group. Then $G$ is not residually finite.

Proof: Suppose $G$ is residually finite. Then $G$ is isomorphic to a subgroup of $\prod_{i \in I} F_{i}$, where $I$ is an index set and each $F_{i}$ is a finite group. Let $p_{i}$ be the projection of $G$ into $F_{i}$. As $G$ is divisible and $F_{i}$ is finite, $p_{i}(G)$ is a finite divisible group and hence is the trivial group. Thus, $G$ is trivial, which is a contradiction. Therefore, $G$ is not residually finite.

From Lemma 3.2, we know for example that $\mathbb{R}$, the additive group of real numbers, is not a residually finite group since it is divisible. However, every free group is residually finite $[4$, p.116].

We now turn to the variety of all $T\left(\aleph_{0}\right)$-groups and we are able to prove the main result of this section.

THEOREM 3.3. Let $\mathfrak{T}_{\aleph_{0}}$ be the variety of all $T\left(\aleph_{0}\right)$-groups and let $\mathfrak{A}_{\aleph_{0}}$ be the variety of all Abelian $T\left(\aleph_{0}\right)$-groups. Then $\left|\mathcal{T}_{\aleph_{0}}\right|_{2}$ and $\left|\mathfrak{A}_{\aleph_{0}}\right|_{2}$ are not varieties of groups.

Proof: By the remark above and Lemma 3.1, $\mathfrak{T}_{\aleph_{0}}$ contains each free group with some Hausdorff group topology. So each free group is in $\left|\mathfrak{T}_{\aleph_{0}}\right|_{2}$. Suppose that $\left|\mathfrak{T}_{\aleph_{0}}\right|_{2}$ is a variety of groups. Then $\left|\mathcal{T}_{\aleph_{0}}\right|_{2}$ must be the variety of all groups, as every group is a quotient of a free group. However, as remarked above, $\mathbb{R}$ is not residually finite and hence by Lemma 3.1 is not contained in $\left|\mathfrak{T}_{\aleph_{0}}\right|_{2}$. Thus, $\left|\mathfrak{T}_{\aleph_{0}}\right|_{2}$ is not a variety of groups. The Abelian case is proved similarly.

\section{4. $T(m)$-GROUPS}

In this section, we shall examine $T(m)$-groups for $m>\aleph_{0}$ and see that there is a proper class of varieties of topological groups $\mathfrak{V}$ such that $|\mathfrak{V}|_{2}$ is not a variety of groups. However, we shall find that there is also a proper class of varieties of topological groups $\mathfrak{V}$ such that $|\mathfrak{V}|_{2}$ is a variety of groups. 
THEOREM 4.1. Let $m$ be an infinite cardinal and let $\mathfrak{T}_{m}$ be the variety of all $T(m)$-groups. Then the class of groups $\left|\mathfrak{T}_{m}\right|_{2}$ is not a variety. of groups.

PROOF: Every free group is a $T\left(\aleph_{0}\right)$-group and therefore is a $T(m)$-group. Suppose $\left|\mathfrak{T}_{m}\right|_{2}$ is a variety of groups. Then $\left|\mathfrak{T}_{m}\right|_{2}$ contains all groups. However, by [2] for example, for each cardinal $m$ there exists a simple group $G$ of cardinality strictly greater than $m$. Thus $G$ is not contained in $\left|\mathfrak{T}_{m}\right|_{2}$ and so $\left|\mathfrak{T}_{m}\right|_{2}$ is not a variety of groups.

Up to this point we have only presented varieties of topological groups $\mathfrak{V}$ where the class of groups underlying Hausdorff groups contained in $\mathfrak{V}$ do not form varieties of groups. However, this is not always the case, as we see in Theorem 4.2.

We shall denote by $\mathbb{Z}\left(p^{\infty}\right)$ the set of all complex numbers $\exp \left(2 \pi i k / p^{n}\right)$, where $p$ is a fixed prime, $k$ runs through all integers, and $n$ through all nonnegative integers. Note that $\mathbb{Z}\left(p^{\infty}\right)$ is a (countable) Abelian group under multiplication.

THEOREM 4.2. Let $m$ be any cardinal number such that $m>\aleph_{0}$ and let $\mathfrak{A}_{m}$ be the variety of all Abelian $T(m)$-groups. Then the class of groups $\left|\mathfrak{A}_{m}\right|_{2}$ is a variety of groups; indeed $\left|\mathfrak{A}_{m}\right|_{2}$ is the variety of all Abelian groups.

Proof: We know that the group of rational numbers $\mathbb{Q}$ under addition is an Abelian group of cardinality $\aleph_{0}<m$. Therefore, $\mathbb{Q}$ with the discrete topology forms a (Hausdorff) $T(m)$-group. Hence, $\mathbb{Q} \in\left|\mathfrak{A}_{m}\right|_{2}$. Similarly, $\mathbb{Z}\left(p^{\infty}\right) \in\left|\mathfrak{A}_{m}\right|_{2}$.

Let $G$ be an Abelian group. Then $G$ can be embedded in a divisible Abelian group $H$ (see, for example, [1, Theorem A.15]). Further, $H$ is isomorphic to a subgroup of a product of copies of $\mathbb{Q}$ and $\mathbb{Z}\left(p^{\infty}\right)$ where $p$ ranges over all primes (see, for example, $[1$, Theorem A.14]). Now, $G$ with the topology induced by the product of copies of $\mathbb{Q}$ and $\mathbb{Z}\left(p^{\infty}\right)$ each with the discrete topology forms a Hausdorff $T(m)$-group. Therefore, $G \in\left|\mathfrak{A}_{m}\right|_{2}$. Thus, $\left|\mathfrak{A}_{m}\right|_{2}$ is the variety of all Abelian groups.

Theorems 3.3, 4.1 and 4.2 lead us to ask the following question.

Open Question. Let $m$ be an infinite cardinal. In the notation of Proposition 2.2, for what varieties of groups $\mathcal{V}$ is $\left|\mathfrak{V}_{m}\right|_{2}$ a variety of groups? When the answer is in the affirmative, is $\left|\mathfrak{V}_{m}\right|_{2}=\mathcal{V}$ ?

\section{REFERENCES}

[1] E. Hewitt and K.A. Ross, Abstract harmonic analysis I (Springer-Verlag, Berlin, Heidelberg, New York, 1963).

[2] G. Higman, 'On finite simple permutation groups', Publ. Math. Debrecen 3 (1954), 221-226.

[3] R.D. Kopperman, M.W. Mislove, S.A. Morris, P. Nickolas, V. Pestov and S. Svetlichny, 'Limit laws for wide varieties of topological groups', Houston J. Math 2 (1996), 307-328. 
[4] W. Magnus, A. Karrass and D. Solitar, Combinatorial group theory: Presentations of groups in terms of generators and relations (Dover Publications Inc., New York, 1976).

[5] S.A. Morris, 'Varieties of topological groups', Bull. Austral. Math. Soc. 1 (1969), 145-160.

[6] S.A. Morris, 'Varieties of topological groups III', Bull. Austral. Math. Soc. 2 (1970), 165-178.

[7] S.A. Morris, Pontryagin duality and the structure of locally compact Abelian groups (Cambridge University Press, Cambridge, 1977).

[8] S.A. Morris, 'Varieties of topological groups - A survey', Colloq. Math. 46 (1982), 147-165.

[9] H. Neumann, Varieties of groups (Springer-Verlag, Berlin, Heidelberg, New York, 1967).

Department of Mathematics

University of Wollongong

Wollongong NSW 2522

Australia

e-mail: caz_mcphail@uow.edu.au 